\title{
Low-dose whole thorax radiation therapy for COVID-19 pneumonia: inpatient onboarding process for a randomized controlled trial
}

\author{
Shearwood McClelland III ${ }^{1}$, Amy C. Miller ${ }^{1}$, Mark D. Williams ${ }^{2}$, Benjamin P. Anthony ${ }^{3}$, Arnab Chakravarti ${ }^{4}$, \\ Paul A. Anthony ${ }^{1}$ \\ ${ }^{1}$ Department of Radiation Oncology, Indiana University School of Medicine, Indianapolis, United States \\ ${ }^{2}$ Department of Medicine, Indiana University School of Medicine, Indianapolis, United States \\ ${ }^{3}$ Department of Otolaryngology, Indiana University School of Medicine, Indianapolis, United States \\ ${ }^{4}$ Department of Radiation Oncology, The Ohio State University, Columbus, United States
}

\begin{abstract}
Background: The mortality of the SARS-CoV-2 virus (COVID-19) has been associated with a pulmonary inflammatory response resulting in hypoxemia and rapid clinical decline. PREVENT is an ongoing prospective multicenter Phase II randomized controlled trial where patients hospitalized with COVID-19 pneumonia are randomized to low dose radiation therapy (RT) versus control (clinicaltrials.gov, NCT04466683). We describe the inpatient onboarding process of the center contributing the largest number of patients to this trial.

Materials and methods: COVID-19 hospital admissions were attained by the clinical research manager and radiation oncologist daily. Text message contact was made with infectious disease, critical care, and nursing staff with reciprocal discussion of the trial protocol and approval for virtual consulting of the patient. Witnessed informed consent was obtained first by telephone and later in person. Simulation and treatment (performed without a computer plan) was performed on a linear accelerator with one personal protective equipment-protected therapist moving in and out of the treatment room, and a second therapist manning the console. Following on-site dose calculation by physics, the radiation oncologist approved the fields prior to treatment delivery.

Results: Between August 28, 2020 and October 6, 2020, the first 10 enrolled patients on this multicenter trial were randomized and treated at our institution; no team member (research staff, radiation oncology) contracted COVID-19 while employing this protocol.
\end{abstract}

Conclusion: This represents the first published protocol to address efficient and safe recruitment of COVID-19 patients for a radiation oncology trial, serving as a model for conducting recruitment of COVID-19 patients for clinical trials.

Key words: COVID-19 pneumonia; radiation therapy; patient recruitment; randomized controlled trial

Rep Pract Oncol Radiother 2021;26(6):1057-1059

\section{Introduction}

Infection with the SARS-CoV-2 virus (COVID-19) results in a protective inflammatory re- sponse, which can result in loss of lung compliance and hypoxemia, manifesting as rapid clinical decline and high mortality rate (3-6\%) [1-3]. Recent work has indicated a potential therapeutic window

Address for correspondence: Shearwood McClelland III, MD, Department of Radiation Oncology, Indiana University School of Medicine, 535 Barnhill Drive, RT 041, Indianapolis, IN 46202, United States; e-mail: drwood@post.harvard.edu

This article is available in open access under Creative Common Attribution-Non-Commercial-No Derivatives 4.0 International (CC BY-NC-ND 4.0) license, allowing to download articles and share them with others as long as they credit the authors and the publisher, but without permission to change them in any way or use them commercially 
for treatment to prevent ventilator-dependence thereby reducing mortality [4-6].

Radiation exposure as high as $1 \mathrm{~Gy}$ (100 cGy) has been previously demonstrated to mitigate inflammation in several conditions including both bacterial and viral pneumonia through modulation of the immune response [7-8]. The hypothesis of the ongoing PREVENT Phase II randomized controlled trial (NCT04466683) is that a single low-dose of radiation therapy ( 0.35 Gy or 1 Gy) can effectively reduce the host inflammatory response associated with COVID-19 pneumonia to objectively improve clinical outcomes by preventing ventilator-dependence; a recent randomized double-blinded study revealed that radiation therapy administration once ventilator-dependence for COVID-19 pneumonia has occurred does not improve outcomes [9]. The following is a description of the inpatient onboarding process of the center contributing the largest number of patients to this ongoing trial.

\section{Materials and methods}

The onboarding process at our institution (Indiana University) began with proactive virtual meetings with key infections disease and emergency medicine physicians at our intra-institutional subsites. Following Institutional Review Board approval as a PREVENT enrollment center (limited initially to 10 patients), our onboarding process began on August 28, 2020.

COVID-19 hospital admissions from the previous day were attained by the clinical research manager each morning, screened for eligibility, and then sent to the designated radiation oncologist who further delineated from this list. Common exclusion criteria were: 1. Oxygen saturation greater than $96 \%$ on room air, 2. Age younger than 50, 3. More than nine days from initial COVID-19 symptomatology. From this list, HIPAA-compliant text message contact was made with infectious disease, critical care, and nursing staff with reciprocal discussion of the trial protocol and approval for consulting the patient. Subsequently, a virtual consult was made with the patient, with witnessed informed consent obtained via telephone, followed later by confirmatory in-person consent by our research associate. Patients were informed of the extremely rare chance of radiation-induced malignancy (particularly given: 1 . Their age $>50$, and 2 . The low 1

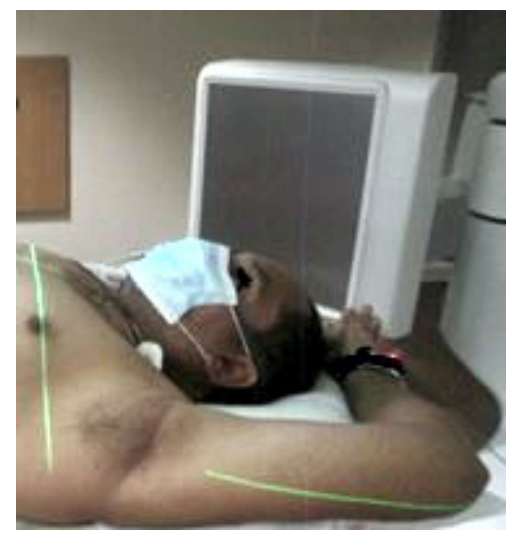

Figure 1. Patient with COVID-19 being treated with lowdose radiation $(0.35 \mathrm{~Gy})$ as per the ongoing PREVENT trial

Gy maximum dose in this trial which is at most $40 \%$ of the dose received by atomic bomb survivors in Japan), and the 3-to-1 randomized protocol of the trial prior to consent [10].

Patients randomized to RT were treated within 24 hours of consent. Simulation and treatment were performed on a linear accelerator with one personal protective equipment-protected therapist moving in and out of the treatment room, and a second therapist manning the console. On-site dose calculation was performed by physics, after which the radiation oncologist approved the fields prior to treatment delivery. Simulation and treatment were performed without a computer plan; therapists underwent several practice sessions prior to treating patients on trial. The average transport time from the patient room to the vault and back to the patient room was 30 minutes total; the average total time of treatment in the vault was 20 minutes. COVID-19 patients were treated as the last patient of the day, with no more than one patient per day being treated (Fig. 1). Surfaces cleaned in the treatment room (wiped with alcohol) included the couch, gantry and nearby fomites. As all patients were treated at the end of the day, a dedicated linac was not required.

\section{Results}

Between August 28, 2020 and October 6, 2020, the first 10 enrolled patients on this multicenter trial were randomized and treated at our institution; no research staff member nor radiation oncology clinical team member contracted COVID-19 while employing this protocol. The success of this protocol has led it to be actively emulated by sev- 
eral contributing centers to the PREVENT trial. Following successful IRB approval to enroll more than the additional 10 patients approved for our institution, our onboarding protocol has continued to safely enroll patients on-trial.

\section{Discussion}

This represents the first published protocol to address efficient and safe recruitment of COVID-19 patients for a radiation oncology trial. Despite the procedural hurdles of conducting a trial in the midst of a pandemic, the combination of recruitment efficiency (approximately 2 patients/week) and safety to the research and clinical teams (no COVID-19 infections) of this protocol has allowed for the PREVENT trial to make substantial progress in meeting recruitment goals $(n=100)$. This onboarding process serves as a model in conducting recruitment of COVID-19 patients for clinical trials, and continues to be worthy of emulation at additional centers.

\section{Conflicts of interest}

No author has any conflicts of interest.

\section{Funding}

This study received research support from Varian. $\mathrm{Dr}$. Chakravarti receives research funding from $\mathrm{Ab}$ bVie, Inc.

\section{Acknowledgments}

The authors would like to thank Sarah Brennan, Kathryn Lauer and Drs. James Jones, Kevin Shiue, Namita Agrawal, and Tim Lautenschlaeger for invaluable assistance.

\section{References}

1. Sanders J, Monogue M, Jodlowski T, et al. Pharmacologic Treatments for Coronavirus Disease 2019 (COVID-19). JAMA. 2020; 323(18): 1824-1836, doi: 10.1001/ jama.2020.6019, indexed in Pubmed: 32282022.

2. Tian S, Hu W, Niu Li, et al. Pulmonary Pathology of EarlyPhase 2019 Novel Coronavirus (COVID-19) Pneumonia in Two Patients With Lung Cancer. J Thorac Oncol. 2020; 15(5): 700-704, doi: 10.1016/j.jtho.2020.02.010, indexed in Pubmed: 32114094.

3. Huang $C$, Wang $Y$, Li X, et al. Clinical features of patients infected with 2019 novel coronavirus in Wuhan, China. Lancet. 2020; 395(10223): 497-506, doi: 10.1016/s01406736(20)30183-5, indexed in Pubmed: 31986264.

4. Zhou F, Yu T, Du R, et al. Clinical course and risk factors for mortality of adult inpatients with COVID-19 in Wuhan, China: a retrospective cohort study. Lancet. 2020; 395(10229): 1054-1062, doi: 10.1016/s0140-6736(20)30566-3, indexed in Pubmed: 32171076.

5. Gong J, Ou J, Qiu X, et al. A Tool for Early Prediction of Severe Coronavirus Disease 2019 (COVID-19): A Multicenter Study Using the Risk Nomogram in Wuhan and Guangdong, China. Clin Infect Dis. 2020; 71(15): 833-840, doi: 10.1093/cid/ciaa443, indexed in Pubmed: 32296824.

6. Richardson S, Hirsch J, Narasimhan M, et al. Presenting Characteristics, Comorbidities, and Outcomes Among 5700 Patients Hospitalized With COVID-19 in the New York City Area. JAMA. 2020; 323(20): 2052, doi: 10.1001/ jama.2020.6775, indexed in Pubmed: 32320003.

7. Oppenheimer A. Roentgen therapy of "virus" pneumonia. Am J Roentgenol Rad Ther. 1943; 49: 635-638.

8. Oppenheimer A. Roentgen therapy of interstitial pneumonia. J Pediatr. 1943; 23(5): 534-538, doi: 10.1016/ s0022-3476(43)80256-0.

9. Papachristofilou A, Finazzi T, Blum A, et al. Low-Dose Radiation Therapy for Severe COVID-19 Pneumonia: A Randomized Double-Blind Study. Int J Radiat Oncol Biol Phys. 2021; 110(5): 1274-1282, doi: 10.1016/j. ijrobp.2021.02.054, indexed in Pubmed: 33677049.

10. Dracham CB, Shankar A, Madan R. Radiation induced secondary malignancies: a review article. Radiat Oncol J. 2018; 36(2): 85-94, doi: 10.3857/roj.2018.00290, indexed in Pubmed: 29983028. 